\title{
Eine Sammlung Lurche und Kriechthiere von Westafrika.
}

\author{
Von \\ Dr. Ant. Reichenow. \\ Hierzu Tafel IX.
}

Meine Reise nach Guinea während der Jahre 72 und 73, auf welcher ich in Begleitung meines verstorbenen Gefährten, Dr. L üh der, einen grossen Theil der Küstendistricte Westafrikas besuchte und an verschiedenen Punkten zoologische Sammlungen anstellte, hat an Reptilien und Lurchen nur geringe Ausbeute geliefert. Wenn ich aber mit nachfolgenden Zeilen, in welchen ich die gesammelten Arten mit den bezüglichen Notizen meines Tagebuches aufführe, auch nicht durch zahlreiche neue Formen die Wissenschaft bereichern kann, so dürften doch einzelne Bemerkungen von Interesse, die Angaben der Fundorte für die Kenntniss der geographischen Verbreitung der einzelnen Arten ein willkommener Beitrag sein.

Die Naturverhältnisse Westafrikas erscheinen für die in Rede stehenden Thiergruppen als vorzugsweise günstige. Durch die starke Verdunstung der zahlreichen grösseren und kleineren Flüsse ist die Luft überall und zu allen Zeiten mit Wasserdunst geschwängert. Die ge-waltigen Niederschläge, welche nur wenige Wochen im Jahre aussetzen, schaffen vereint mit den glühenden Strahlen der Sonne ein Pflanzenleben, wie es üppiger 
nicht gedacht werden kann. Dichter Urwald, von Wasserstrassen häufig durchschnitten, hin und wieder freiere Steppengegend, mit mannshohem Grase und zerstreuten Büschen und Bäumen bedeckt, Lagunen oder Binnenteiche einschliessend, bilden im Grossen und Ganzen den landwirthschaftlichen Charakter Westafrikas ${ }^{1}$ ). Dürre Ebenen fehlen fast vollständig; überall die gleiche Fülle der Vegetation: Verhältnisse, welche höchst günstige Lebensbedingungen für die Kriechthiere und Lurche bieten. Die Thatsächlichkeit entspricht jedoch nicht diesen Voraussetzungen. Nach dem gegenwärtigen Stande unserer Kenntniss erscheint der kleine Süden Afrika's artenreicher als der Westen, obwohl letzerer, abgesehen von der weiteren Ausdehnung, den scheinbar günstigeren Naturverhältnissen, durch die in der Regel an Mannigfaltigkeit der Formen reicheren äquatorialen Districte jenem bevorzugt ist.

Von Batrachiern sind die Laubfrösche am zablreichsten vertreten, dürftig die Teichfrösche. In der Froschmusik entbehren die westafrikanischen Landschaften einen der schönsten Reize. Nur selten vernimmt man das Gequak einzelner Frösehe oder dumpfe Krötenlaute. Niemals hörte ich die so einförmigen und doch so angenehm harmonischen Concerte, welche in unseren Frühlingsabenden und Nächten den Naturbeobachter zu entzücken vermögen. Die Kriechthiere entziehen sich durch ihre Lebensweise sehr den Blicken des Reisenden. Als gewöhnliche Erscheinungen fallen in den Ortschaften der Neger die an einigen Stellen zahlreichen Agamen (colonorum), an anderen Euprepes-Arten auf. Auch den Gecko, Hemidactylus guineensis, findet man hier immer. In der Steppenlandschaft begegnet man häufig der Brillenschlange. In den Flüssen sind Crokodile zahlreich; auch Warans und Lederschildkröten sieht man bäufig. Die Fortpflan-

1) In einem demnāchst im Journal für Ornithologie (Juli-Heft 1874) erscheinenden Aufsatze habe ich die Naturverhāltnisse Westafriks, soweit sie für das Thierleben von Bedeutung sind, ausführlicher behandelt, worauf ich verweise. 
zung, die Paarung der Batrachier und Reptilien fällt in den Beginn der Regen, also je nach der Lage der betreffenden Oertlichkeit nördlich oder südlich vom Aequator, in unsere Sommer- oder Wintermonate, doch beobachtete ich auch mehrmals Schlangen in der Paarung während der Trockenmonate.

Zum Folgenden ist noch zu bemerken, dass die Farbenangaben sich auf lebende Individuen beziehen.

\section{Amphibia.}

Fam. Aglossa.

Dactylethra Mülleri Pet.

Ooerseite hellolivenbraun; Unterseite gelblichweiss; Krallen schwarz. Das grösste der gesammelten Exemplare misst $10 \mathrm{Ctm}$. vom Kopf bis zur Zehenspitze.

Die Thiere sind ungemein beweglich und flink. Wir erhielten eine Anzahl derselben aus einem kleinen Tümpel am Camerunflusse. Das so constatirte Vorkommen am Camerun dürfte wohl die nördlichste Verbreitung dieses Frosches sein.

Fam. Bufones.

Bufo guineensis Schl.

Oberseite halbgraubraun mit schwarzen Flecken, Unterseite schmutzigweiss. Erreicht 17 bis $18 \mathrm{Ctm}$. Länge.

Die gemeinste und verbreitetste Kröte in Westafrika, vertritt in den südlicheren Districten den nur in den nördlichsten vorkommenden pantherinus. Wir fanden sie häufig an der Goldküste und am Camerun, in Gebäuden und in der Steppe als Bewohner alter Termitenhügel.

\section{Fam. Ranae.}

Rana Bibroni Hall.

Oben hellgraubraun mit schwarzen Flecken, auf den Schenkeln zwei hellgrüne Längsstreifen, die im Alkohol gelb werden, Unterseite weiss.

Am Camerun gefunden. Die gesammelten Exemplare messen vom Kopf bis zur Zehenspitze $13 \mathrm{Ctm}$.

Rana occipitalis Güntb.

Archiv f. Naturg. $\mathrm{XXXX}$. Jahrg. 1. Bd. 
Oberseite erdbraun mit schwarzer Marmorirung; Unterseite weiss mit grauen Flecken; Seiten des Körpers und der Füsse weissgelb mit grauen, an den Schenkeln schwarzen Flecken. Erreicht $32 \mathrm{Ctm}$. Länge. Bei Accra an der Goldküste fanden wir diesen Frosch häufig in einer Lagune und in kleinen Binnenteichen. Er lebt nach Art unseres grünen Teichfrosches, hält sich meistens im Wasser auf, kommt selten und nur auf kurze Zeit ans Land.

Fam. Hylae.

Petropedetes n. gen.

Zunge länglich herzförmig, hinten frei, ausgekerbt; Gaumenzähne hinter den Nasenlöchern, den hintern Rand derselben nicht berührend und einander mehr genähert als diese; Zehenscheiben flach, aber ausgeprägt; Finger frei; Zehen durch kurze Schwimmhäute verbunden; Paukenfell deutlich.

Hinsichtlich der Zungenbildung und der Stellung der Gaumenzähne stimmt diese neue Gattung mit der Gattung Platymantis (vergl. Günther Cat. Batr. Sal. pag. 93) überein, unterscheidet sich von derselben aber durch die ausgeprägteren Zehen- und Fingerscheiben und die Schwimmhäute zwischen den Zehen.

Petropedetes cameronensis Rchw. n. sp. Taf. IX, Fig. II a. b.

Zehen durch kurze Schwimmhäute verbunden, welche bei den beiden ersten Zehen bis an das Nagelglied reichen; von der fünften $1 \frac{1}{2}$, von der dritten 2 , von der vierten 3 Glieder frei; dritte Zehe wenig länger als fünfte, vierte bedeutend länger; Finger frei, Folge derselben: 1. 2.4.3; Haut der Oberseite feinkörnig, auf dem Rücken wenige in vier undeutliche Längsreihen gestellte Drüsen; Haut der Unterseite glatt; oben rostbraun mit schwarzer Zeichnung, unten weiss. Länge vom Kopf bis zur Zehenspitze $12 \mathrm{Ctm}$.

Wurde an einem Gebirgsbache bei Bimbia, in den Vorbergen des Camerun gefangen, wo die Thiere zahlreich auf den Felsen umherhüpften. 
Leptopelis rufus Rchw. n. sp. Taf. IX. Fig. I a. b. Finger durch kurze Schwimmbäute verbunden, welche zwei Glieder frei lassen; Folge der Finger: 1. 2. 4. 3; Zehen bis an die Nagelglieder verbunden, nur von der vierten zwei Glieder frei; füfte Zehe kaum länger als dritte, vierte bedeutend länger: Zunge herzförmig, hinten frei und ausgekerbt; Gaumenzähne zwischen den beiden Nasenlöehern, dieselben nicht berührend; Haut der Oberseite sehr fein granulirt, ebenso die Kehle und Unterseite der Extremitäten; Brust und Bauch stärker gekörnt; oben einfarbig rotbbraun, unten weiss, auf den Körperseiten und Unterseiten der Schenkel dunkel gefleckt, unter jeder Achsel ein runder dunkler Fleck. Länge $12 \mathrm{Ctm}$.

In der Zungenbildung, der Stellung der Gaumenzähne wie in der ganzen Gestalt ist diese Art sehr ähnlich der Aubryi Schleg., unterscheidet sich von derselben aber durch die stärkeren Schwimmhäute zwischen den Fingern und durch die Färbung.

Gefunden wurde die Art von uns im Walde bei Victoria, am Fusse der Camerunberge.

\section{Reptilia.}

\section{Ophidia.}

Fam. Typhlopidae.

Onychocephalus Kraussi Jan.

Ein grosses Exemplar von $60 \mathrm{Ctm}$. Länge in den Bergen von A guapim an der Goldküste gesammelt.

Unterseite gelbbraun; Oberseite dunkelgoldbraun mit zwölf Längsreihen gelbbrauner Flecke. Die einzelnen Schuppen haben gelbbraunen Saum und dunkelen Mittelfleck an der Basis. Nach anhaltendem Regen findet man die Blödaugen nicht selten auf den Wegen umherkriechen. Eingehenderes über die Lebensweise konnten wir auch durch die Eingeborenen nicht erfahren.

Onychocephalus liberiensis Hallow.

Gelbbraun, auf der Oberseite mit unregelmässigen, dunkel goldbraunen Flecken. 
Wir fanden diese Art sehr häufig in Aquapim. Es möchte dieselbe wohl nur eine Varietät von Kraussi sein.

Fam. Dendrophidae.

Hapsidrophis smaragdinus Boie.

An der Goldküste und in der Camerungegend angetroffen. Oberkörper grün; Oberkieferrand und Unterkörper weiss, an den Seiten eine grüne Längslinie; ein Strich durch das Auge schwarz. Im Alkohol wird die grüne Farbe blau. Länge der gesammelten Exemplare $90 \mathrm{Ctm}$.

Ahaetulla irregularis Leach.

An der Goldküste und in der Camerungegend nicht selten. In Accra fanden wir diese Schlange oft in den Gebäuden. Oberseite grün, zuweilen mit schmutzig gelbgrünen Flecken; Unterseite gelb. Die einzelnen Rückenschuppen haben einen weissen Randfleck am unteren Saume, der bei gewöhnlicher Lage der Schuppen, am lebenden Thiere, nicht sichtbar ist; die Haut zwischen den Schuppen ist schwarz. Im Alkohol verwandelt sich die grüne Farbe in blau.

Das grösste Exemplar, welches ich mass, hatte eine Länge von $90 \mathrm{Ctm}$.

Fam. Dryophidae.

Cladophis Kirtlandi Dum.

Am Gabun gesammelt, wo diese schöne Schlange oftmals in die Gebäude kommt.

Oberkopf und Kopfseite griin; Kehle und Oberkieferrand weiss; der übrige Körper von unbestimmt grünlichgrauer Farbe, braun marmorirt. Die Brust ist blasser, der Bauch intensiver braun marmorirt. Iris goldgelb. Länge 1,1 Mtr.

Fam. Lycodontidae.

Heterolepis poënsis Smith.

An Camerun gefunden.

Oben schwarz, unten weiss, mit Perlmutterglanz.

Länge des einen gesammelten Exemplares 1 Mtr.

Boodon unicolor Boie. 
An der Goldküste und am Camerun gesammelt. Lg. $60 \mathrm{Ctm}$.

Boodon nigrum Fisch.

Am Camerun gefunden. Länge $70 \mathrm{Ctm}$.

\section{Fam. Elapidae.}

\section{Causus rhombeatus Wagl.}

Diese weit verbreitete Schlange sammelte ich in mehreren jüngeren Exemplaren am Gabun.

Die Färbung ist aschgrau mit schwarzen, grösseren, V-förmigen und kleineren Flecken auf dem Rücken; Unterseite blasser.

Naja haje L.

Sehr häufig im Galande an der Goldküste und auch in der Camerungegend. Ich fand mehrmals Exemplare von etwa 2 Meter Länge, alle von dunkler Färbung: Oberseite schwarz; Schnauzenspitze hornbraun; Kopfseite, Kinn und Kehle gelblich; Brust breit schwarz und gelb quergebändert; übrige Unterseite schwarz.

Die Brillenschlangen bewohnen die gemischte Steppe; im diehten Walde kommen sie nicht vor. In der Nittagshitze kriechen sie, wie die Puffottern, gern auf die Wege, um sich zu sonnen. Stösst man dann plötzlich auf sie, so richten sie sich steil empor, zischen, blasen den Hals auf und speien eine Flüssigkeit auf die Entfernung von mehreren Fussen auf den Ruhestörer, wobei sie nach den Augen zu zielen scheinen. Die Quantität dieser Flüssigkeit, wohl Speichel und jedenfalls mit dem Gifte der Zahndrüsen gemischt, ist ziemlich bedeutend, da die Thiere oft dreimal hintereinander speien, und schliesslich der Saft vom Maule herabtropft. Nach Angabe der Missionäre an der Goldküste, sowie der Eingeborenen, erfolgt Erblindung, wenn jener Saft in das Auge kommt. Zufolge analoger, an Klapperschlangen gemachter Erfahrungen, welche mir die Herren Effeldt und Wagen$\mathrm{füh} \mathrm{r}$ in Berlin mittheilten, scheint es mir indessen zweifelhaft, dass solcher Speichel, bezüglich Schlangengift, eine andere Wirkung auf die Hornhaut ausübe als irgend welche ätzende Flüssigkeit. 


\section{Fam. Viperidae.}

Atractaspis Bibroni Smith.

Ein Exemplar am Camerun gesammelt. Lg. $65 \mathrm{Ctm}$.

Echidua arietans Merrem.

Die Puffoter, diese giftigste der afrikanischen Schlangen, trafen wir vielfach an der Goldküste. Am Camerun ist sie mir nicht vorgekommen.

Wie die Brillenschlange meidet sie den Wald; die gemischte Steppe sagt ihr zu. Während des Tages ruht sie unter dichtem Gestrüppe; nur zur Mittagszeit kriecht sie wohl herror auf eine kahle Stelle, auf die schmalen durch das Gras führenden Fusssteige, um sich an den glühenden Strahlen der Tropensonne zu erfreuen. Wie wohlthuend solche Glühbitze den Schlangen ist, zeigt die Munterkeit dieser bei Tage im Allgemeinen trägen Nachtthiere in solchen Verhältnissen. Ich stiess mehrmals auf Puffottern, welche zusammengerollt sich behaglich sonnten. Aber kaum dass ich sie gesehen, verschwanden die gestörten Thiere pfeilschnell im Grase.

Ein von Negern erlegtes und uns gebrachtes Exemplar mass 1,6 Mtr.

\section{Sauria.}

Fam. Scinci.

Euprepes Reichenowi Pet.

Diese von mir in den Camerunbergen gesammelte Art wurde von Herrn Prof. Peters als neu erkannt und in den Monatsberichten der Kgl. Academie der Wissenschaften zu Berlin (Jahrg. 1874 Februar) unter vorstehendem Namen beschrieben. Ich fand die Art nur in den Bergen, während ich in der Ebene an der gleichen Lokalität die nachfolgende Art aussehliesslich antraf.

Euprepes breviceps Pet.

Oberseite kupferbraun, längs des Rückens vier Reihen kleiner weisser oder gelblicher Flecke, die mehr oder wẻniger deutlich von dunkelbraunen Flecken umsäumt sind; Unterseite weiss. Die Färbung ändert $a b$; die beiden äusseren Fleckenreihen des Rückens sind 
oft undeutlich. Die Länge der ausgewachsenen Individuen beträgt 12 bis $15 \mathrm{Ctm}$.

Diese Art ist sehr gemein in der Camerungegend und südlich bis zum Gabun. In den Ortschaften, an den Hütten der Eingeborenen, in den Plantagen und Feldern sind sie anzutreffen und leben ganz nach Art der Eidechsen. Sie halten sich ausschliesslich auf dem Boden auf und klettern nicht, wie die Agamen, auf die Dächer der Hütten oder auf Bäume.

\section{Fam. Agamae.}

Agama colonorum Daud.

Sehr häufig an der Goldküste, weniger zahlreich in den südlicheren Küstendistricten bis zum Gabun.

Ich fand diese Agame in den Ortschaften oder in den Feldern und Plantagen in der Nähe derselben. Im Walde dagegen traf ich die nachstehend beschriebene Varietät. In Accra war jede Lehmmauer, die Wände und Dächer der Negerhütten und die Bäume mit diesen Thieren geschmückt. Ich sage geschmückt, denn die Männchen mit ibren feuerrothen Köpfen und Schwanzenden gewähren in der That einen prächtigen Anblick. Lauernd sitzen sie in der Sonne, mit den Köpfen nickend, bei jeder auffallenden Erscheinung den Oberkörper auf und abwärts bewegend, und schiessen pfeilschnell auf die erspähte Beute, wie sie überhaupt ungemein schnell in ihren Bewegungen sind.

Das alte $\delta$ im Prachtkleide hat feuerrothen Kopf, Kehle gelb gesprenkelt; Körper und Beine glänzend dunkel stahlblau, über dem Kü̈cken ein heller Längsstrich, der bisweilen fehlt; Unterseite des Schwanzes vom After bis zur Mitte strohgelb, die entsprechende Oberseite an der Basis hellstahlblau, dann feuerroth, die zweite Hälfte des Schwanzes dunkelstahlblau.

Vorstehende Beschreibung nach einem Exemplare vom Camerun. Bei solchen von der Goldküste fehlt die strohgelbe Färbung an der Unterseite des Schwanzes. Der letztere ist an der Basalhälfte (oben und unten) hellstahlblau; darauf folgt das feuerrothe Band, welches nur 
eine kurze dunkelstahlblaue Spitze übrig lässt. Sie erreichen eine Länge von $35 \mathrm{Ctm}$. Das Weibchen ist hellbraun mit hellerer Rückenlinie und dunkleren Flecken auf dem Rücken; Unterseite blasser; Kehle weisslich, dunkel marmorirt.

Die Jungen gleichen den Weibchen, haben aber hellgelbe Flecken auf Kopf und Nacken.

In den Bergen von Aquapim fand ich eine schöne Varietät dieser Art, welche ich immer nur in Waldlichtungen auf Bäumen antraf. Bei den Männchen derselben war der Kopf und das sonst rothe Schwanzband rein weiss.

Im Alkohol gehen die schönen Farben der männlichen Exemplare vollständig verloren.

Fam. Geckones.

Hemidactylus guineensis Pet.

Schmutzig fleischfarben, Oberseite braun marmorirt. Gemein an allen von mir besuchten Punkten Westafrikas.

Erreicht eine Länge von $12 \mathrm{Ctm}$.

Fam. Monitores.

Varanus niloticus Dum. Bib.

Dieser über ganz Afrika verbreitete Waram war gemein an allen von mir besuchten Punkten, in Lagunen und Flüssen. Um die Mittagszeit sieht man die Thiere häufig ausserhalb des Wassers auf den Wurzeln oder Aesten der Mangrove sitzen und sich sonnen. Die Nahrung besteht vorzugsweise in Fischen; doch fressen sie auch Säugethiere. Gefangene nahmen Ratten uud Mäuse sehr begierig.

Die Färbung ändert hinsichtlich der Zeichnung vielfach ab. Im Allgemeinen ist die Oberseite schwarz, bei Alten schwarzbraun, mit Querreihen oft abgezirkelt runder, gelber Flecke; auf dem Kopfe feine gelbe Zeichnung; Unterseite weissgelb mit schwarzen oder schwärzlichen Querbinden; Schwanz schwarz mit breiten, gelben Querbinden oder aus einzelnen Flecken gebildeten Querreihen. Ich sah Exemplare von 1,5 Mtr. Lg. 


\section{Fam. Chamaeleontes.}

Chamaeleo senegalensis Cuv.

Von der Goldküste.

Die Färbung ist im gewöhnlichen Zustande grau braun mit schwarzer Seitenbänderung.

Chamaeleo dilepis Leach.

Eine sehr häufige Art am Gabun.

In der Ruhe ist die Farbe der Thiere hellgrün mit dunkleren Streifen, welche über die Seiten vom Rücken auslaufen; Brustkamm weiss; Iris hellgelb bis rothbraun.

Wird das Chamäleon gereizt, so ändert sich die grüne Farbe in grau; später erscheinen schwarze Punkte und Streifen; der Kehlsack wird gelbbraun mit schwarzen Punkten. Im Zustande höchster Erregung des jähzornigen Geschöpfes ist dasselbe völlständig schwarz; zuweilen erscheinen dann auf den Körperseiten noch kleine gelbe Flecken.

Chamaeleo cristatus Stutch.

Am Camerun und Gabun nicht häufig.

Chamaeleo Owenii Gray.

Nur einmal in Victoria am Fusse der Camerunberge erhalten.

\section{Fam, Crocodilina}

Crocodilus cataphractus Cuv.

Gemein im Camerundelta, wie im oberen Flusse, sehr häufig im Wuri. Im Gabun erhielt ich die Art ebenfalls.

Vielfach erhielt ich den Beweis, dass die Krokodile im seichten Wasser den Menschen und so jedenfalls auch grössere Thiere nicht angreifen. Eine Furth, welche durch eine Lagune bei Accra führte, wurde beständig von den Negern benutzt, obwohl die Krokodile zahlreich in dem Wasser waren, und niemals war ein Unglücksfall vorgekommen. Ebenso sah ich die Eingeborenen im Wuri an seichten Stellen ohne Furcht vor den allenthalben sichtbaren Krokodilen baden. Mehrfach dagegen kam es daselbst während unserer Anwesenheit vor, dass Neger an tiefen Stellen des Flusses sogar aus den Canoes von Krokodilen weggeschnappt wurden, indem die Thiere plötzlich aus 
dem Wasser hervorschossen, den Mann beim Arme oder am Beine ergriffen und hinunterzogen. Jedenfalls greifen die Thiere deshalb nicht gern im seichten Wasser an, weil das Opfer hier Widerstand leisten kann, während sie dasselbe im tiefen Wasser sofort ertränken. Das schmackhafte Fleisch der Krokodile wird von den Eingeborenen sehr geschätzt.

\section{Chelonia.}

Fam. Trionychidae.

Trionyx aegyptiacus Geoffr.

In einer Lagune bei Accra gefangen und später, wahrscheinlich diese Art, im Camerundelta bemerkt.

Fam. Chelydidae.

Pentonyx gabonensis Dum.

Vom Camerun.

Diese Art scheint nur geringe Grösse zu erreichen. Lg. des Rückenschildes $7 \mathrm{Ctm}$.

Fam. Chersemydae.

Cinixys erosa Gray.

Nicht selten am Camerun. Wir sammelten nur jüngere Exemplare.

\section{Erklärung der Abbildungen.}

Tafel IX.

Fig. I. Leptopelis rufus: Geöffnetes Maul, zeigt die Zungenbildung und Stellung der Gaumenzähne.

a. Hand. b. Fuss.

Fig. II. Petropedetes cameronensis:

Ganze Figur. a. Geöffnetes Maul. b. Fuss. 


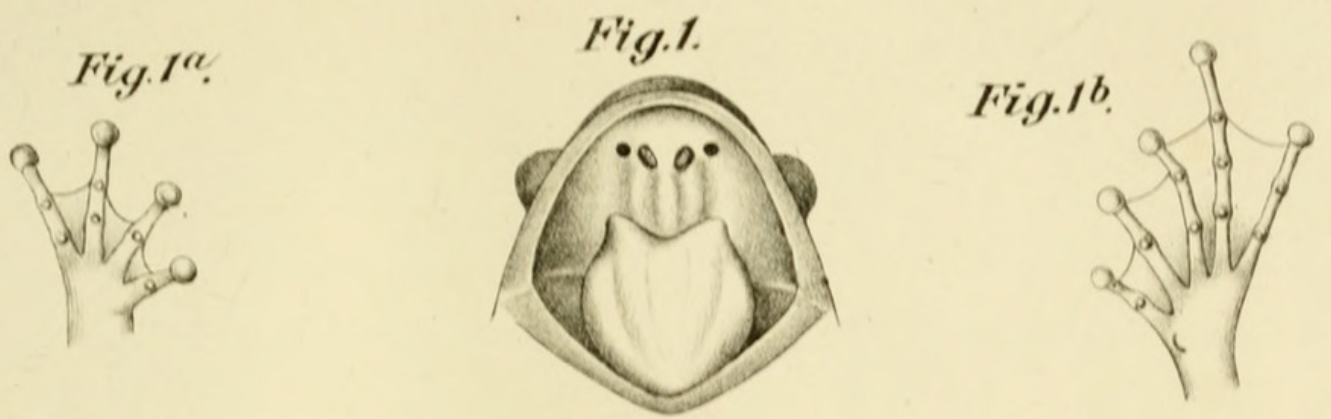

Fig. 2. nat.Cr:

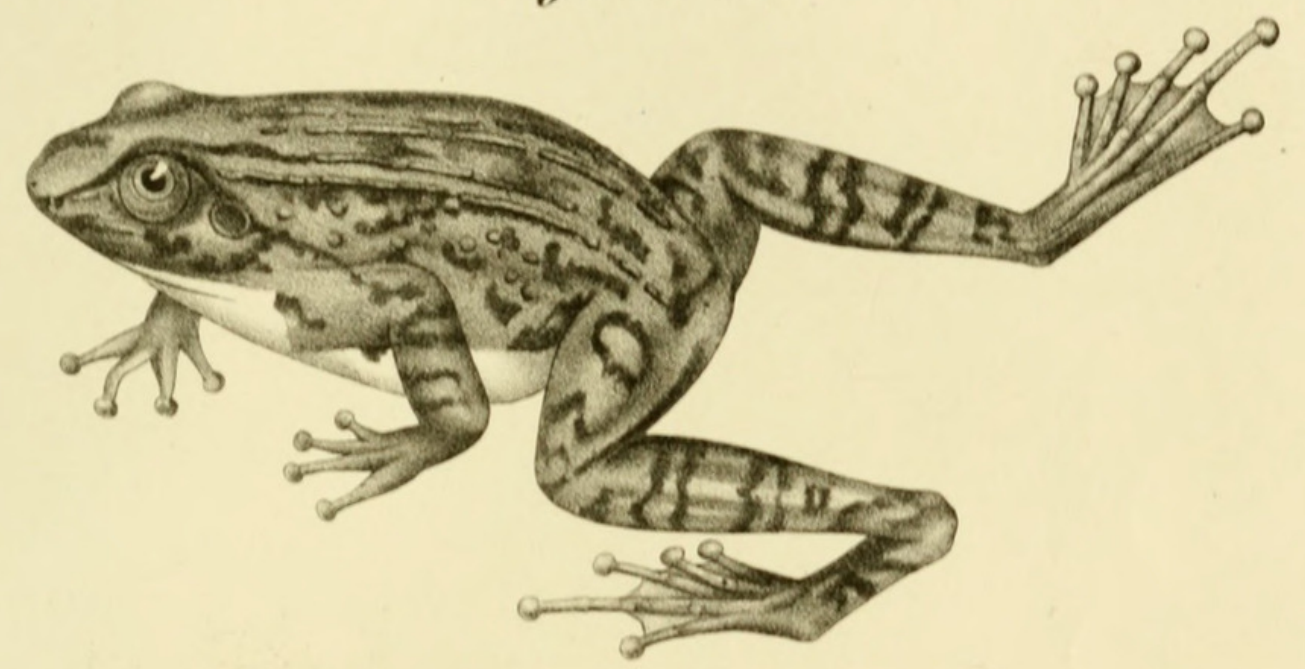

Fig. $2 a$.

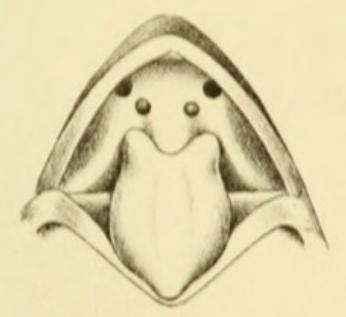

Fig. 'b

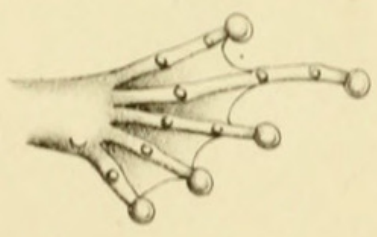




\section{$2 \mathrm{BHL}$ Biodiversity Heritage Library}

Reichenow, Anton. 1874. "Eine Sammlung Lurche und Kriechthiere von Westafrika." Archiv für Naturgeschichte 40, 287-298.

https://doi.org/10.5962/bhl.part.10802.

View This Item Online: https://www.biodiversitylibrary.org/item/29638

DOI: https://doi.org/10.5962/bhl.part.10802

Permalink: https://www.biodiversitylibrary.org/partpdf/10802

\section{Holding Institution}

MBLWHOI Library

Sponsored by

MBLWHOI Library

\section{Copyright \& Reuse}

Copyright Status: NOT_IN_COPYRIGHT

This document was created from content at the Biodiversity Heritage Library, the world's largest open access digital library for biodiversity literature and archives. Visit BHL at https://www.biodiversitylibrary.org. 